\title{
Primary Prophylaxis to Prevent Tuberculosis Infection in Prison Inmates: A Randomized, Double- Blind, Placebo-Controlled Trial
}

\author{
Roberto Dias de Oliveira, ${ }^{1,2}$ Andrea da Silva Santos, ${ }^{3}$ Cassia Barbosa Reis, ${ }^{1,2}$ Alessandra de Cássia Leite, ${ }^{2}$ \\ Flávia Patussi Correia Sacchi, ${ }^{4}$ Rafaele Carla Pivetta de Araujo, ${ }^{3}$ Paulo César Pereira dos Santos, ${ }^{3}$ Valeria Cavalcanti Rolla, ${ }^{5}$ \\ Leonardo Martinez, ${ }^{6}$ Jason Andrews, ${ }^{6}$ and Julio Croda $^{2,7 *}$ \\ ${ }^{1}$ State University of Mato Grosso do Sul, Dourados, Brazil; ${ }^{2}$ Federal University of Mato Grosso do Sul, Campo Grande, Brazil; ${ }^{3}$ Federal University of \\ Grande Dourados, Dourados, Brazil; ${ }^{4}$ Municipal Health Office, Dourados, Brazil; ${ }^{5}$ Oswaldo Cruz Foundation, Rio de Janeiro, Brazil; ${ }^{6}$ Stanford \\ University, Stanford, California; ${ }^{7}$ Oswaldo Cruz Foundation, Campo Grande, Brazil
}

\begin{abstract}
In many low- and middle-income countries, tuberculosis (TB) incidence in prisons is high, exposing incarcerated populations to an elevated risk of TB infection. We conducted a randomized, double-blind, placebo-controlled trial among HIV-negative male inmates of a high TB burden prison to determine whether isoniazid given twice weekly $(900 \mathrm{mg})$ for 12 months prevents TB infection. The primary outcome was QuantiFERON-TB Gold in Plus (QFT) conversion to $\geq 0.35$ international units per milliliter (IU/mL) at 6 months; secondary outcomes included alternative QFT thresholds $(\geq 0.7, \geq 2.0$, and $\geq 4.0 \mathrm{IU} / \mathrm{mL})$. In total, 467 participants were randomly assigned to intervention $(N=258)$ or control $(N=209)$. In an interim analysis of participants who had completed 6 months of follow-up $(N=170)$, QFT conversion occurred in $20.8 \%(19 / 91)$ and $21.5 \%$ (17/79) of participants in intervention and control arms (efficacy: $2.9 \%, P=0.91$ ), respectively. The trial was then stopped according to the trial protocol, and the remaining participants prematurely discontinued. In an analysis of secondary outcomes, the intervention arm had significantly lower rates of conversion at a cutoff of $\geq 2.0 \mathrm{IU} / \mathrm{mL}$ (efficacy: $82.6 \%, P<0.01$ ). In conclusion, $900 \mathrm{mg}$ of isoniazid, administered twice a week, did not effectively prevent QFT conversion at a cutoff point $\geq 0.35 \mathrm{IU} / \mathrm{mL}$ in a trial of QFT-negative inmates. Higher QFT cutoffs are associated with sustained conversion and greater protection. Future clinical trials that evaluate protection for latent infection should use the highest cutoff than that recommended by the manufacturer.
\end{abstract}

\section{INTRODUCTION}

Tuberculosis (TB) is an urgent health crisis among incarcerated populations globally. ${ }^{1,2}$ In low-and middle-income countries, incidence rates of TB in prisons are reported to be 33 times higher than those in the general population. ${ }^{3,4}$ These disparate disease risks are driven by several factors including high rates of alcohol and drug abuse, poor ventilation, crowding, and elevated rates of comorbidities such as HIV, hepatitis $\mathrm{B}$ and $\mathrm{C}$, and diabetes. ${ }^{5,6}$ These combinations of risk factors lead to an extraordinary high force of infection in prisons; for example, in three recent tuberculin-negative cohorts of prisoners from Brazil, Colombia, and Iran, between $15 \%$ and $25 \%$ annually converted their skin test. ${ }^{7-9}$

Previous interventions in prisons have concentrated on treating latently infected prisoners to prevent disease ${ }^{10}$ or mass screening of entire prisons, providing treatment to those with disease. ${ }^{9}$ These interventions have had minimal success. Because of the elevated TB transmission rates in prisons, interventions that do not directly prevent transmission may not interrupt the disease cycle in areas of heavy TB burden. ${ }^{11}$

Prisoners in Brazil have short incarceration terms, usually leaving prison within 1-2 years. ${ }^{12}$ At entry into prison, less than $20 \%$ of prison inmates have latent TB. ${ }^{13}$ Therefore, an intervention that protects prisoners from acquiring infection during imprisonment may be adequate for long-term TB control in these prisons. Although there is a large body of empirical evidence demonstrating that isoniazid prevents progression to active TB, ${ }^{14,15}$ whether isoniazid prevents primary infection is unclear. In phase IV, a randomized, double-blind, placebocontrolled study in a prison with a high TB burden, we evaluated

*Address correspondence to Julio Croda, Oswaldo Cruz Foundation, 92 Gabriel Abrão Street, Campo Grande 79074-460, Brazil. E-mail: julio.croda@fiocruz.br the safety and efficacy of isoniazid given twice a week to prevent latent TB infection. Our primary outcome was QuantiFERONTB Gold in Plus (QFT) conversion to $\geq 0.35$ international units per milliliter (IU/mL) at 6 months; secondary outcomes included alternative QFT thresholds ( $\geq 0.7, \geq 2.0$, and $\geq 4.0 \mathrm{IU} / \mathrm{mL}$ ).

\section{MATERIALS AND METHODS}

Study design. This randomized, double-blind, placebocontrolled, phase IV clinical trial was performed at Dourados State Penitentiary in Mato Grosso do Sul, Brazil. From September 2017 to July 2018, we enrolled male inmates aged between 18 and 45 years serving time in Dourados State Penitentiary with a negative QFT test and sputum culture. We excluded all participants testing QFT positive (antigen-nil $\geq 0.35$ $\mathrm{IU} / \mathrm{mL}$ for either tube), previously treated for TB or currently with TB, with a history of epilepsy or current epilepsy, HIV positive, hepatitis B or C positive, or demonstrating serum concentrations of glutamic-oxaloacetic transaminase (SGOT) or serum concentrations of glutamic-pyruvic transaminase (SGPT) three times greater than the upper limit and the Alcohol Use Disorders Identification Test ${ }^{16}$ greater than or equal to 15 points.

In the first 3 months of recruitment, we included individuals with a negative QFT test as defined by the manufacturer $(<0.35 \mathrm{IU} / \mathrm{mL})$. However, after 3 months of enrollment, the eligibility criterion was changed from any QFT result less than $0.35 \mathrm{IU} / \mathrm{mL}$ to any QFT result less than $0.20 \mathrm{IU} / \mathrm{mL}$ based on emerging evidence that QFT positivity thresholds require reevaluation. ${ }^{17-19}$

The study protocol was approved by our Institutional Review Board (\#2.758.659, August 2016, State University of Mato Grosso do Sul, Dourados, Brazil). All participants provided written informed consent, and study data were collected and managed using electronic data capture tools. No participant with positive QFT accessed for study eligibility 
was treated for latent TB because the use of this test as a criterion for treating latent TB was not incorporated into the Brazilian TB guideline; for those with documented QFT conversion, latent TB treatment with $300 \mathrm{mg}$ of isoniazid daily was offered.

Randomization and blinding. Participants were randomized into two arms, intervention and control, using 1:1 allocation. We performed a simple randomization in a single block with 728 elements using alphanumeric codes, generated by the principal investigator. For each participant, the unblinded study pharmacist assigned one of these codes, which placed it in one arm. No other team member (including laboratory staff) had access to the code identification key, thus ensuring continuity of blindness. The codes were only opened in case of proven need, for example, clinical management of a severe adverse event (AE). Blinding occurred immediately following randomization. Specifically, after randomization, the pharmacist prepared identical bottles containing isoniazid or placebo and referred them to the field staff. The vials were identified by labels with the participant's name, the study identification number, and the randomization code. The placebo used was identical to isoniazid in appearance and was produced by the same industry that produced isoniazid.

Study outcomes. The planned primary study outcome was QFT conversion at the 12-month visit, defined as an interferon- $y$ value $\geq 0.35 \mathrm{IU} / \mathrm{mL}$, for either tube. However, because the trial was stopped early, we analyzed QFT conversion at the 6-month visit. As a secondary analysis, we analyzed QFT conversion at alternative interferon-gamma value cutoffs $(\geq 0.7, \geq 2.0$, and $\geq 4.0 \mathrm{IU} / \mathrm{mL})$ were performed, based on recent evidence linking high quantitative QFT values and disease. ${ }^{17-19}$

We used definitions from the Guideline by International Conference Harmonization ${ }^{20}$ for adverse events and severe AEs (SAEs). Adverse event severity was assessed using a toxicity table from the Common Terminology Criteria for AEs ${ }^{21}$ and the grouping in body systems according to the Medical Dictionary for Regulatory Activities. We defined hepatotoxicity as SGOT or SGPT > 5 times the normal and the presence of symptoms of hepatitis such as unexplained anorexia, nausea, vomiting, dark urine, icterus, rash, persistent paresthesia of the hands and feet, persistent fatigue, and weakness or fever of more than 3 days/or abdominal tenderness, especially right upper quadrant discomfort. ${ }^{22,23}$

Study discontinuation was defined as participants who decided to stop participating in the study (because of participant withdrawing consent) but remained in the prison. "Transfer or release from prison" was defined as participants released from prison, through either transfer to another prison or receiving parole.

Study activities. The intervention arm received $900 \mathrm{mg}$ of isoniazid orally twice weekly under directly observed therapy (DOT). The control arm received placebo under the same conditions. All participants received two weekly visits, which, in addition to DOT, included questioning and observation of signs and symptoms of TB and AE.

Additional visits were performed in the third month of followup, in which participants were clinically evaluated and had a blood sample collected for SGOT and SGPT testing. In addition to clinical evaluations, liver enzyme testing, QFT, and sputum culture were performed in the sixth month and at the termination of the study for those who had not reached 6 months of follow-up.
Statistical analysis. We estimated from previous studies ${ }^{12}$ that 560 individuals would be required to achieve $90 \%$ power to detect a $50 \%$ difference in QFT conversion between the intervention group and the control group. The level of significance was set at $5 \%$. Accounting for a $30 \%$ loss to follow-up, the enrollment goal was 728 individuals.

After recruitment of at least 100 participants in the sixth month, an interim analysis was performed by an independent data and safety monitoring board. We performed this evaluation to verify the feasibility of continuity of the study and adequacy of the sample calculation. If the primary outcome difference between arms was less than $2.5 \%$, then discontinuation of the study was recommended.

For $\mathrm{AE}$, we calculated the percentage using the number of participants who received at least one dose, and for severity, we used the total number of events recorded.

The outcomes are reported as percentages when converted, and group comparisons are reported as intervention efficacy (1-RR, where RR is the relative risk) with Cls. $P$-values were estimated using a two-sample test for equivalence of proportions, and statistical significance was set at $P<0.05$. For all expected values less than 5 , Fisher's exact test was used. Otherwise, chi-square significance testing was used. For continuous variables, two-sided $t$-tests and the MannWhitney rank-sum test were used for independent samples. The Shapiro-Wilk test was applied to analyze the distribution of the samples, and the Mann-Whitney rank sum test was used if $P<0.05$. Analyses were performed with SPSS 25 (IBM SPSS, Armonk, NY) and SAS 9.4 (SAS Institute Inc., Cary, NC).

\section{RESULTS}

From September 2017 to June 2018, we recruited 1,097 men to assess study eligibility. Most exclusions were due to a positive QFT result (46.2\%). Four hundred sixty-seven participants underwent randomization (Figure 1). The demographic and clinical characteristics of individuals allocated to the two arms of the study were statistically similar (Table 1), despite differential allocation between arms. This difference between arms occurred because we generated the randomization codes in a single block with 728 elements, and, initially, the allocation to the intervention arm was by chance greater than that to the control arm. However, in the group used in the final analysis, we did not observe any difference between the arms (intervention arm, 53.5\%; 95\% Cl: 46.0, 60.8 and control arm, 46.4\%; 95\% Cl: 39.1, 53.9). Follow-up time was equivalent in the intervention and control arms (mean of 180 days in both arms, $P=0.798$ ).

The sample was predominantly young men (median age: 28 years; interquartile range [IQR]: 23,34$)$. More than half of them were smokers $(59.3 \%)$ or used marijuana, and almost all were Bacillus Calmette-Guérin (BCG) vaccinated (92.7\%). Twothirds of participants were previously incarcerated, and one in four reported comorbidities. Participants lived in overcrowded cells (median: 13 cellmates; IQR: 7,15 ).

Initially, 104 doses over 52 weeks were planned for each participant (corresponding to 12 months of intervention), but with the interruption of the study after the interim analysis, a median of 60 doses were taken (IQR: 53, 62).

Discontinuations and losses to follow-up were high in both arms (intervention, 126/258 [48.8\%]; control, 87/209 [41.6\%], $P=0.14$ ), and the primary reason for study interruptions in 


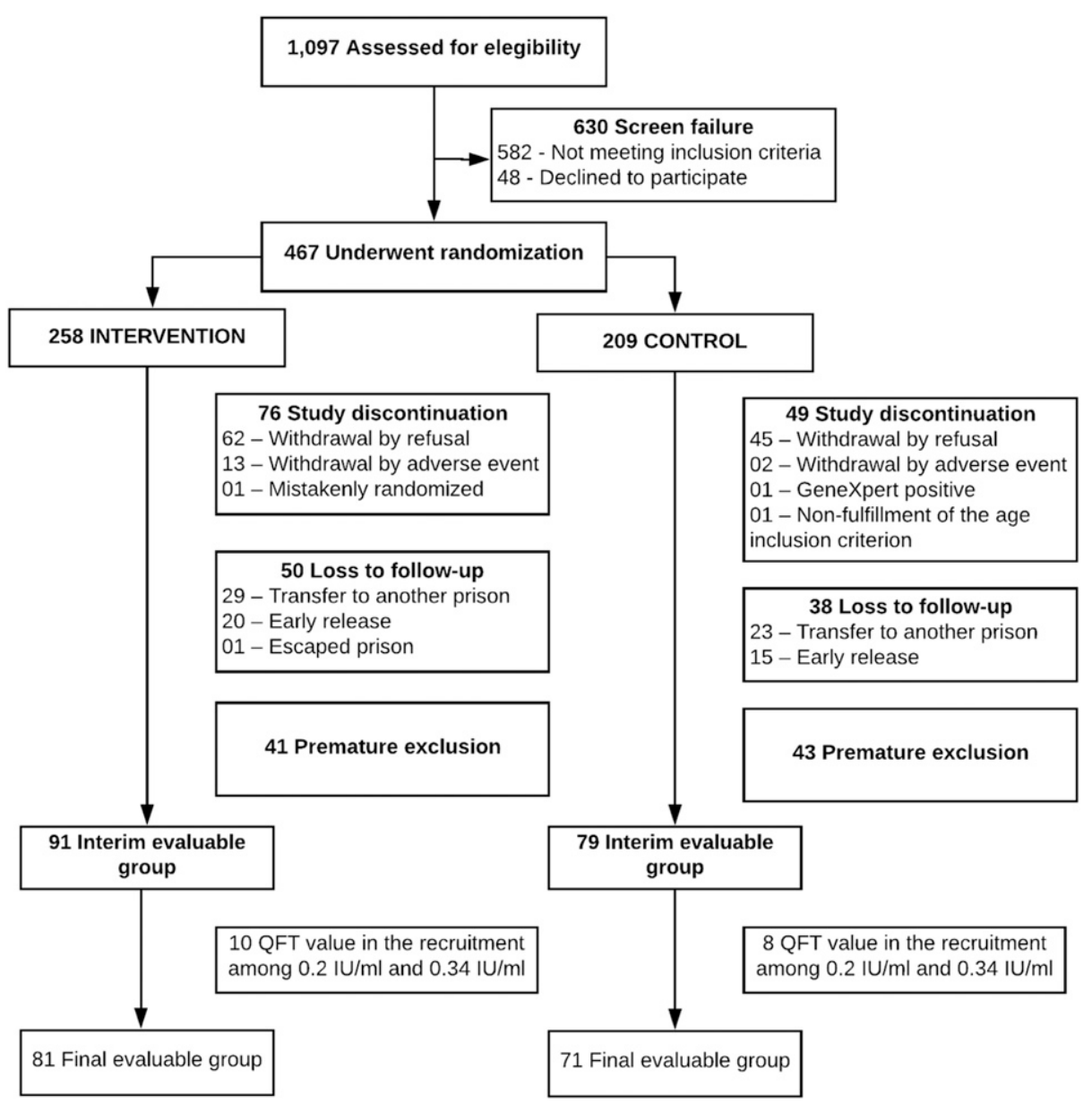

FIGURE 1. Flowchart of study population, randomization, and analyses of study outcomes.

both arms was participant elective withdrawal (24.0\% versus $21.5 \%, P=0.60)$, followed by transfer or release from prison (19.0\% versus $18.2 \%, P=0.92)$, and withdrawal following $\mathrm{AE}$ (5.0\% versus $1.0 \%, P=0.02)$.

In June 2018, 170 participants were included in the interim analysis at 6 months from baseline (6-month outcome); 91 in the intervention arm (53.5\%) and 79 in the control arm (46.5\%) (Table 2). QuantiFERON-TB Gold in Plus conversion occurred in 19 of the 91 participants $(20.8 \%)$ in the intervention arm, compared with 17 of the 79 participants $(21.5 \%)$ in the control arm (absolute risk difference: $0.7 \%$, efficacy: $2.9 \%, P=0.91$ ). Based on the study protocol, the study was discontinued at this time because the absolute risk difference between arms was less than $2.5 \%$. Eighty-four participants were prematurely discontinued of the follow-up, 41 in the intervention arm, and 43 in control arm. After study discontinuation, we performed another analysis with participants who underwent QFT at the 6-month visit (Table 2). In a post hoc analysis excluding 18 participants with a baseline QFT value between 0.2 and 0.34 $\mathrm{IU} / \mathrm{mL}$, the efficacy was greater $(38.1 \%, P=0.16)$. Among these 18 participants, 7 (38.8\%) showed conversion at 6 months (considering QFT $\geq 0.35 \mathrm{IU} / \mathrm{mL}$ ), and $2(11.1 \%)$ with a cutoff $\geq 0.7 \mathrm{IU} / \mathrm{mL}$. Those who have not converted $(61.1 \%)$ showed a reduction in the QFT mean value observed at enrollment, in one or both tubes (TB1 $0.14 \mathrm{UI} / \mathrm{mL}$ versus $0.08 \mathrm{UI}$ / $\mathrm{mL}, P=0.21$ and TB2 $0.24 \mathrm{UI} / \mathrm{mL}$ versus $0.10 \mathrm{Ul} / \mathrm{mL}, P=0.15$ ).
We also conducted an analysis of secondary outcomes with alternative QFT cutoff values (Table 2). Using a QFT cutoff of $\geq 2.0 \mathrm{lU} / \mathrm{mL}$, the intervention arm conversion rate was lower than the control arm conversion rate both in the interim analysis at 6 months (2/91 [2.1\%] versus 10/79 [12.6\%]; efficacy: $82.6 \%, P<$ 0.01 ) and in the secondary analysis excluding 18 participants with a baseline QFT value between 0.2 and $0.34 \mathrm{IU} / \mathrm{mL}(1 / 81$ [1.2\%] versus 10/71 [14.1\%]; efficacy: $92.1 \%, P<0.01$ ). When using other QFT cutoff points ( $\geq 0.7$ and $\geq 4.0 \mathrm{lU} / \mathrm{mL}$ ), there was a trend toward a lower conversion rate in the intervention arm, but this did not reach statistical significance (Table 2).

The intervention group had lower QFT values at 6 months when compared to the control group in both tubes [(Mean \pm SD) TB1 $0.25 \pm 0.79 \mathrm{lU} / \mathrm{mL}$ versus $0.53 \pm 1.25 \mathrm{IU} / \mathrm{mL}, P=0.88$; TB2 $0.23 \pm 0.54 \mathrm{IU} / \mathrm{mL}$ versus $0.49 \pm 1.21 \mathrm{IU} / \mathrm{mL}, P=0.308$.

Of the 467 participants who received at least one dose, 218 (46.7\%) had no AE, 142 (30.4\%) had one AE, and 107 (22.9\%) had more than one $A E$ (Figure 2). There was a nonsignificantly higher proportion of $A E$ reported in the intervention arm than in the control arm (30.2\% versus $23.0 \%, P=0.26)$. Gastrointestinal disorders $(P=0.004)$, hepatobiliary disorders $(P=$ $0.005)$, nervous system disorders $(P=0.0009)$, and general disorders and administration site conditions $(P=0.005)$ were higher in the isoniazid (INH) arm, whereas respiratory, thoracic, and mediastinal disorders $(P=0.008)$ were higher in the control arm. Individuals in the intervention arm were more 
TABLE 1

Demographic characteristics of trial arms by randomization and complete evaluable group

\begin{tabular}{|c|c|c|c|c|c|c|}
\hline \multirow{3}{*}{$\begin{array}{l}\text { Characteristic } \\
\text { Total individuals in each arm }\end{array}$} & \multicolumn{3}{|c|}{ Randomization $(N=467)$} & \multicolumn{3}{|c|}{ Evaluable final group $(N=170)$} \\
\hline & \multirow{2}{*}{$\frac{\text { Intervention, } n(\%)}{N=258}$} & \multirow{2}{*}{$\frac{\text { Control, } n(\%)}{N=209}$} & \multirow[b]{2}{*}{$P$-value } & \multirow{2}{*}{$\frac{\text { Intervention, } n(\%)}{N=91}$} & \multirow{2}{*}{$\frac{\text { Control, } n(\%)}{N=79}$} & \multirow[b]{2}{*}{$P$-value } \\
\hline & & & & & & \\
\hline Age (years), mean $( \pm S D)$ & $29.2(6.7)$ & $29.0(7.1)$ & $0.41^{*}$ & $30.1(6.1)$ & $29.5(7.2)$ & $0.57^{*}$ \\
\hline \multicolumn{7}{|l|}{ Age-group (years) } \\
\hline $18-29$ & $146(56.6)$ & $122(58.4)$ & 0.69 & $43(47.3)$ & 47 (59.5) & 0.11 \\
\hline 30-39 & 89 (34.5) & $64(30.6)$ & 0.37 & $42(46.2)$ & $20(25.3)$ & $<0.01$ \\
\hline $40-45$ & $23(8.9)$ & $23(11.0)$ & 0.45 & $6(6.6)$ & $12(15.2)$ & 0.07 \\
\hline \multicolumn{7}{|l|}{ Race/color } \\
\hline White & $130(50.4)$ & $109(52.2)$ & 0.70 & $42(46.2)$ & $35(44.3)$ & 0.81 \\
\hline Mixed & $105(40.7)$ & $90(43.1)$ & 0.60 & 39 (42.9) & $38(48.1)$ & 0.49 \\
\hline Black & $22(8.5)$ & $9(4.3)$ & 0.06 & $10(11.0)$ & $5(6.3)$ & 0.30 \\
\hline Asian & $1(0.4)$ & $1(0.5)$ & $>0.99 \dagger$ & $0(0.0)$ & $1(1.3)$ & - \\
\hline Body mass index, mean $( \pm S D)$ & $24.7(3.5)$ & $24.9(3.6)$ & $0.53^{\star}$ & $24.6(3.7)$ & $24.2(3.2)$ & $0.33^{*}$ \\
\hline$\leq 18.5$ & $43(16.7)$ & 29 (13.9) & 0.40 & $5(5.5)$ & $2(2.5)$ & 0.36 \\
\hline $18.6-24.9$ & 188 (72.9) & 151 (72.3) & 0.88 & $49(53.8)$ & $52(65.8)$ & 0.11 \\
\hline $25-29.9$ & $23(8.9)$ & $25(12.0)$ & 0.28 & $30(33.0)$ & $21(26.6)$ & 0.37 \\
\hline$\geq 30$ & $4(1.6)$ & $4(1.9)$ & $>0.99$ & $7(7.7)$ & $4(5.1)$ & 0.51 \\
\hline Less than 8 years of education & $157(60.9)$ & $135(64.6)$ & 0.40 & $57(62.6)$ & $55(69.6)$ & 0.34 \\
\hline BCG vaccine scar & 237 (91.9) & $196(93.8)$ & 0.42 & $82(90.1)$ & 75 (94.9) & 0.25 \\
\hline Smoker & $157(60.9)$ & $120(57.4)$ & 0.45 & $57(62.6)$ & $41(51.9)$ & 0.16 \\
\hline Comorbidities & 54 (20.9) & $46(22.0)$ & 0.77 & $18(19.8)$ & $17(21.5)$ & 0.78 \\
\hline Other medications & 32 (12.4) & $23(11.0)$ & 0.64 & $7(7.7)$ & $8(10.1)$ & 0.58 \\
\hline \multicolumn{7}{|l|}{ Use of drugs } \\
\hline Marijuana & $160(62.0)$ & $127(60.8)$ & 0.78 & $52(57.1)$ & $48(60.8)$ & 0.63 \\
\hline Cocaine & $82(31.8)$ & 75 (35.9) & 0.35 & 32 (35.2) & $34(43.0)$ & 0.29 \\
\hline Other & $10(3.9)$ & $8(3.8)$ & 0.97 & $2(2.2)$ & $3(3.8)$ & 0.57 \\
\hline Prior incarceration & $173(67.1)$ & $131(62.7)$ & 0.32 & 67 (73.6) & 55 (69.6) & 0.56 \\
\hline Mean no. of incarcerations $( \pm S D)$ & $2.3(1.6)$ & $2.2(1.6)$ & $0.89^{\star}$ & $2.3(1.8)$ & $2.3(1.7)$ & $0.87^{*}$ \\
\hline Mean prisoners per cell $( \pm S D)$ & $11.3(5.0)$ & $11.8(5.0)$ & $0.96^{\star}$ & $10.4(4.7)$ & $11.4(5.3)$ & $0.20^{*}$ \\
\hline External visit & $135(52.3)$ & $128(61.2)$ & 0.05 & 47 (51.6) & 47 (59.5) & 0.31 \\
\hline
\end{tabular}

likely to develop dizziness $(P=0.06)$, nausea $(P=0.004)$, malaise $(P=0.001)$, and an elevated SGPT $(P=0.04)$.

Early withdrawal attributable to an $\mathrm{AE}$ occurred in 13 participants in the intervention arm and in two participants in the control arm $(5.0 \%$ [95\% Cl: $2.9,8.5]$ versus $1.0 \%$ [95\% Cl: $0.03,3.6], P=0.01)$. Causes that motivated the participant to withdraw in the intervention arm included gastric pain $(N=1)$, low back pain $(N=1)$, somnolence $(N=2)$, itching $(N=2)$, elevated SGOT/SGPT $(N=1)$, headache $(N=3)$, malaise $(N=$ $1)$, diarrhea $(N=1)$, vomiting $(N=1)$, heartburn $(N=1)$, and dizziness $(N=1)$. In the control arm, causes that motivated early withdrawal were low back pain $(N=1)$, dysuria $(N=1)$, headache $(N=1)$, and gastric pain $(N=1)$.
Permanent discontinuation of treatment was recommended by medical staff in two SAE (one in each study arm), both related to asymptomatic grade 3 SGPT, identified in the third month of follow-up. No death, hospitalization, or active TB was recorded in this clinical trial.

\section{DISCUSSION}

There is an urgent need for effective tools to prevent TB infection in high-transmission settings such as prisons, mines, and health facilities. Although isoniazid and other TB drugs have proven efficacious in the prevention of disease by the treatment of latent infection, it is unknown whether primary infection can be

TABLE 2

QuantiFERON conversions, by trial arm, and efficacy of isoniazid as primary prevention

\begin{tabular}{|c|c|c|c|c|c|c|}
\hline \multirow[b]{2}{*}{ Outcome } & \multirow[b]{2}{*}{ Study population } & \multirow{2}{*}{$\begin{array}{l}\text { QuantiFERON conversion } \\
\text { threshold (IU/mL) }\end{array}$} & Intervention arm & Control arm & \multirow[b]{2}{*}{ Efficacy, \% $(95 \% \mathrm{Cl})$} & \multirow[b]{2}{*}{$P$-value } \\
\hline & & & Conversion events, $n(\%)$ & Conversion events, $n(\%)$ & & \\
\hline \multirow[t]{5}{*}{ Six months QFT conversion } & All participants with & $\geq 0.35$ & $\begin{array}{c}N=91 \\
19(20.8)\end{array}$ & $\begin{array}{c}N=79 \\
17(21.5)\end{array}$ & $2.9(-73.4,45.7)$ & 0.91 \\
\hline & 6 months follow-up & $\geq 0.7$ & $9(9.8)$ & $11(13.9)$ & $28.9(-62.5,68.9)$ & 0.42 \\
\hline & & $\geq 2.0$ & $2(2.1)$ & $10(12.6)$ & $82.6(23.1,96.0)$ & $<0.01$ \\
\hline & & $\geq 4.0$ & $1(1.0)$ & $4(5.0)$ & $78.3(-90.1,97.5)$ & 0.16 \\
\hline & & & $N=81$ & $N=71$ & & \\
\hline \multirow[t]{4}{*}{ Six months QFT conversion } & Participants with baseline & $\geq 0.35$ & $12(14.8)$ & $17(23.9)$ & $38.1(-20.5,68.2)$ & 0.16 \\
\hline & $\mathrm{QFT}<0.20 \mathrm{IU} / \mathrm{mL}^{*}$ & $\geq 0.7$ & $7(8.6)$ & $11(15.4)$ & $44.2(-36.1,77.1)$ & 0.20 \\
\hline & & $\geq 2.0$ & $1(1.2)$ & $10(14.1)$ & $91.2(33.2,98.8)$ & $<0.01$ \\
\hline & & $\geq 4.0$ & $1(1.2)$ & $4(5.6)$ & $78.0(-91.5,97.4)$ & 0.16 \\
\hline
\end{tabular}

$\mathrm{IU} / \mathrm{mL}=$ international units per milliliter; QFT = QuantiFERON-TB Gold in Plus .

*Excludes 18 participants who we randomized with a QFT value between 0.2 and $0.34 \mathrm{IU} / \mathrm{mL}$. 


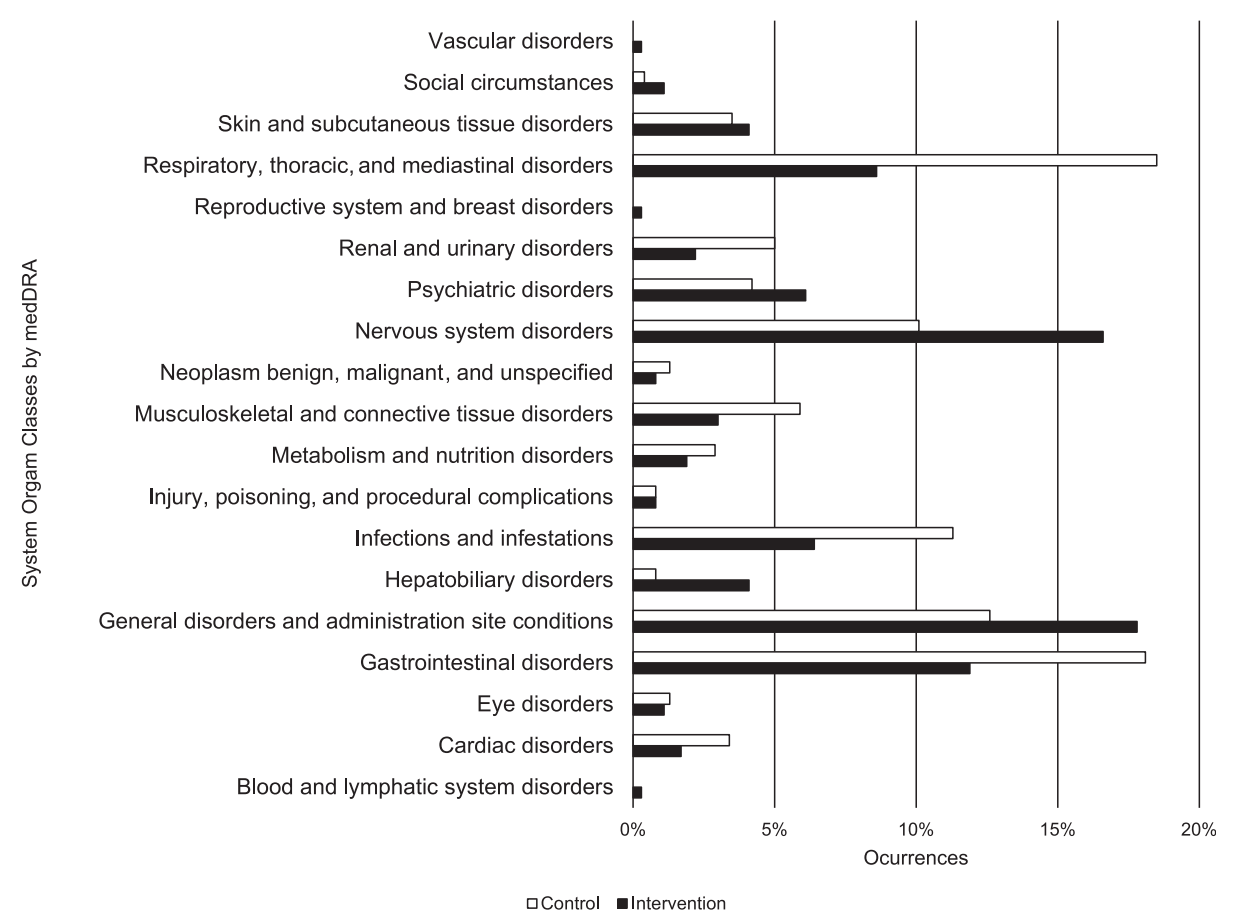

FIGURE 2. Adverse events by system organ classes by the Medical Dictionary for Regulatory Activities stratified by intervention (black) and control (white) arms.

prevented by pre-exposure chemotherapy. The present trial was stopped early because of the lack of efficacy $(2.9 \%, P=0.91)$ to prevent QFT conversion at the prespecified criteria. At analysis, excluding 18 participants who had negative QFT between 0.20 and $0.34 \mathrm{IU} / \mathrm{mL}$ at inclusion revealed a $38.1 \%$ reduction in QFT conversion at any cutoff $\geq 0.35 \mathrm{IU} / \mathrm{mL}(P=0.16)$. In an analysis of secondary outcomes, we found a large efficacy $(82.6 \%, P<0.01)$ of INH against QFT conversion when using a cutoff $\geq 2.0 \mathrm{IU} / \mathrm{mL}$.

We believe the reason for this discrepancy was most likely because of the change in the study enrollment criteria from QFT $<0.35$ to QFT $<0.20$ that occurred after 3 months of the study. This change was made before unblinding, due to emerging evidence about "uncertainty" or "instability" of QFT results between 0.20 and 0.34 , where conversions and reversions frequently occur because of technical or intraindividual biological variability. ${ }^{17}$ Using a stricter eligibility criterion in a randomized trial should not differentially bias results but reduces classification error. We believe that the inclusion of individuals with $0.20-0.34 \mathrm{IU} / \mathrm{mL}$ biased results toward the null, as evidenced by greater effects seen in those with baseline QFT $<0.20$. Despite this major limitation of the trial, we may nevertheless learn something about the ability of TB drugs to prevent against primary infection.

Two previous clinical trials have assessed the efficacy of isoniazid to prevent conversion to a positive tuberculin skin test. 24,25 In 1963, more than 700 household contacts were assigned to isoniazid and placebo arms, 300 of whom were tuberculin negative and assessed for subsequent tuberculin conversion. ${ }^{25} \mathrm{After}$ 3 years of follow-up, $22.8 \%$ and $15.1 \%$ of tuberculin conversions occurred in the placebo and isoniazid arms, a statistically significant difference. In the 1950s-1960s, the U.S. Public Health Service trials conducted and assessed the efficacy of isoniazid to prevent TB infection and disease in hospitalized mental patients, schools, and household contacts of new and known TB cases. ${ }^{24}$
Among these groups, results were mixed; statistically different rates of 12-month tuberculin conversion were seen among household contacts of new TB cases (13.2\% versus $10.4 \%$ in placebo versus isoniazid arms) and in schools (4.0\% versus $2.6 \%$ in placebo versus isoniazid arms), but no differences were seen in mental patients and contacts of known TB cases. These heterogeneous results suggest other factors, such as statistical power or the study population, may be important to consider when assessing the efficacy of isoniazid to prevent new TB infection. Together with these studies, our results indicate that isoniazid should be further explored as a potential intervention to prevent TB infection in populations at high risk for TB exposure.

Current Latent TB infection regimens are shorter than the INH regimen that we studied. However, since inmates are "at risk" during the entire time they spend in prison, we believe that the longer INH regimen is preferable in this situation.

Our results are consistent with other those of recent studies demonstrating that using the manufacturer-recommended interferon- $\gamma$ value cutoff of $\geq 0.35 \mathrm{lU} / \mathrm{mL}$ for conversion may lead to high numbers of false-positive results. ${ }^{17-19,26,27}$ Several recent studies have demonstrated that most individuals who convert their QFT with a value between 0.35 and $0.70 \mathrm{IU} / \mathrm{mL}$ revert to negative on repeat testing. ${ }^{18,28,29}$ Other recent results have suggested similar non-sustainable results at interferon- $\gamma$ values close to the recommended $0.35 \mathrm{IU} / \mathrm{mL}$ cutoff. A recent trial assessing the ability of BCG revaccination to protect against QFT conversion found no efficacy in protection against any QFT conversion but high efficacy of $45.4 \%$ for "sustained conversion" as well high QFT conversions (>4.0 IU/mL). ${ }^{27}$ Other recent longitudinal studies have shown that high cutoffs are much more likely to predict disease progression. ${ }^{18,19}$ Given the extensive evidence base, a more nuanced interpretation of QFT results is advisable, especially in the context of serial testing and in low-risk individuals. ${ }^{26}$ 
In a post hoc analysis using other cutoff points $(0.7,2.0$, and $4.0 \mathrm{IU} / \mathrm{mL}$ ), we observed a large protective effect against QFT conversion of INH with a cutoff point $\geq 2.0 \mathrm{IU} / \mathrm{mL}$ (82.6\%), with a significant difference between the arms. Studies show that high cutoffs are much more likely to predict disease progression, ${ }^{17-19}$ although they increase positive predictive value for incident TB and reduce sensitivity. ${ }^{30}$

The adoption of higher QFT cutoff points does not account for variability in QFT results over time, potentially interfering in diagnostic interpretation. ${ }^{31,32}$ We attempted to minimize temporal variability of the QFT by using an interval of 120 days between tests. We acknowledge that, ideally, a repetition of a positive QFT test to confirm a "sustained" conversion would be best. However, as the study was stopped early, attempting to measure "sustained" conversion was not possible.

The high discontinuation observed in both arms is a concern. Losses to follow-up due to transfer or release from prison (40\% of all discontinuations in the INH arm), although problematic for the study, are not intervention failures because the goal of this intervention is to provide short-term protection against infection in this high-risk environment. If scaled up as an intervention, isoniazid would be discontinued upon release from prison as the exposure is then removed. Among those remaining in prison, we found that $36 \%$ discontinued therapy in the intervention arm (and 28\% in the control arm). Although this rate of discontinuation is high, it is consistent with that of studies of isoniazid when given for treatment of latent TB, for which treatment completion rates are often $<50 \%$. When given to healthy individuals without latent infection, for whom benefit was unknown before this trial, it is not surprising that discontinuation rates were high in both arms. In addition, studies with multiple visits, such as ours, seem to present a higher proportion of dropout by the participant. ${ }^{33,34}$

The isoniazid dose was based on pharmacokinetic results from previous studies and label recommendations. ${ }^{35-37} \mathrm{Al}-$ though the total number of AEs reported in our study was greater than that reported in other studies, ${ }^{10,38,39}$ we hypothesize that more AEs may be reported in participants receiving DOT. At every study visit, participants were asked about the occurrence of an $\mathrm{AE} .{ }^{40}$ Adverse event reporting was nonsignificantly greater in the intervention arm. Adverse events related to the gastrointestinal system were the most common associated with $\mathrm{INH}$ use, corroborating previous trials. ${ }^{41}$ The occurrence of hepatotoxicity was lower than expected based on previous reports. ${ }^{42}$ The high dose of isoniazid was associated with more reported AEs such as dizziness, elevated SGPT, malaise, and nausea.

The interpretation of the trial findings is limited by the loss to follow-up and interruption of the study. With the early interruption of the study, the arms presented differential group allocation, with $11 \%$ more participants in the intervention arm than in the control arm. This may have biased participant randomization but not study blindness. In addition, despite this difference, the groups remained similar in all measured characteristics, indicating any bias was negligible if present.

In conclusion, in this trial, $900 \mathrm{mg}$ of isoniazid, administered twice weekly, did not effectively prevent QFT conversion at a cutoff point $\geq 0.35 \mathrm{IU} / \mathrm{mL}$. Despite the premature interruption of the study, we cannot rule out INH as primary prophylaxis because in QFT conversions with a cutoff $\geq 2.0 \mathrm{lU} / \mathrm{mL}$, the efficacy was $82.6 \%$. Further exploration of INH as a prevention tool is needed using higher QFT cutoffs.
Received February 9, 2020. Accepted for publication June 22, 2020. Published online August 31, 2020.

Acknowledgments: We thank the State Agency of Administration of the Penitentiary System of the State of Mato Grosso do Sul (AGEPEN) for having authorized the study; the direction of the prison "State Penitentiary of Dourados (PED)" for guaranteeing our researchers access to the premises of the establishment, as well as providing space for the field activities; and the Vice Presidency of Research and Biological Collections of the Oswaldo Cruz Foundation for the clinical monitoring of data from this study through the Clinical Research Platform.

Financial support: This work was supported by the National Council for Scientific and Technological Development (Process 420672/20176 and Process 401824/2016-0).

Disclosure: The Institute of Drug Technology (FARMANGUINHOS/ FIOCRUZ) donated isoniazid and placebo; the State Office of Health of Mato Grosso do Sul donated rapid tests for HIV, and hepatitis B and C; and Qiagen donated a portion of the interferon-gamma release assays.

Authors' addresses: Roberto Dias de Oliveira and Cassia Barbosa Reis, School of Nurse, State University of Mato Grosso do Sul, Dourados, Brazil, and Doctorate Program in Infectious and Parasitic Diseases, Federal University of Mato Grosso do Sul, Campo Grande, Brazil, E-mails: roberto@uems.br and cassia@uems.br. Andrea da Silva Santos and Rafaele Carla Pivetta de Araujo, Doctorate Program in Health Sciences, Federal University of Grande Dourados, Dourados, Brazil, E-mails: andrea.santos.enf@gmail.com and rafaele_carla@yahoo.com.br. Alessandra de Cássia Leite, School of Nurse, State University of Mato Grosso do Sul, Dourados, Brazil, E-mail: aleleite17@gmail.com. Flávia Patussi Correia Sacchi, Municipal Tuberculosis and Leprosy Program, Municipal Health Office Dourados, Dourados, Brazil, E-mail: fpatussi@gmail.com. Paulo César Pereir dos Santos, Research Laboratory in Health Sciences, Federal University of Grande Dourados, Dourados, Brazil, E-mail: paulo.cesar.pds@gmail.com. Valeria Cavalcanti Rolla, National Institute of Infectious Diseases Evandro Chagas, Oswaldo Cruz Foundation, Rio de Janeiro, Brazil, E-mail: valeria.rolla@ini.fiocruz.br. Leonardo Martinez and Jason Andrews, Division of Infectious Diseases and Geographic Medicine, School of Medicine, Stanford University, Stanford, CA, E-mails: chopotin@gmail.com and jasonandr@ gmail.com. Julio Croda, Doctorate Program in Infectious and Parasitic Diseases, Federal University of Mato Grosso do Sul, Campo Grande, Brazil, and Oswaldo Cruz Foundation, Campo Grande, Brazil, E-mail: julio.croda@fiocruz.br.

\section{REFERENCES}

1. Telisinghe $L$ et al., 2016. HIV and tuberculosis in prisons in subSaharan Africa. Lancet 388: 1215-1227.

2. O'Grady J, Maeurer M, Atun R, Abubakar I, Mwaba P, Bates M, Kapata N, Ferrara G, Hoelscher M, Zumla A, 2011. Tuberculosis in prisons: anatomy of global neglect. Eur Respir J 38: 752-754.

3. Baussano I, Williams BG, Nunn P, Beggiato M, Fedeli U, Scano F, 2010. Tuberculosis incidence in prisons: a systematic review. PLoS Med 7: e1000381.

4. Bourdillon PM, Gonçalves CCM, Pelissari DM, Arakaki-Sanchez D, Ko Al, Croda J, Andrews JR, 2017. Increase in tuberculosis cases among prisoners, Brazil, 2009-2014. Emerg Infect Dis 23: 496-499.

5. Urrego J, Ko Al, da Silva Santos Carbone A, Paião DSG, Sgarbi RVE, Yeckel CW, Andrews JR, Croda J, 2015. The impact of ventilation and early diagnosis on tuberculosis transmission in Brazilian prisons. Am J Trop Med Hyg 93: 739-746.

6. Dolan K et al., 2016. Global Burden of HIV, viral hepatitis, and tuberculosis in prisoners and detainees. Lancet 388: 1089-1102.

7. Mamani M, Mahmudian H, Majzoobi MM, Poorolajal J, 2016. Prevalence and incidence rates of latent tuberculous infection in a large prison in Iran. Int J Tuberc Lung Dis 20: 1072-1077.

8. Arroyave L, Keynan Y, López L, Marin D, Arbeláez MP, Rueda ZV, 2017. Negative latent tuberculosis at time of incarceration: identifying a very high-risk group for infection. Epidemiol Infect 145: 2491-2499. 
9. Paião DSG et al., 2016. Impact of mass-screening on tuberculosis incidence in a prospective cohort of Brazilian prisoners. BMC Infect Dis 16: 533.

10. White MC, Tulsky JP, Lee JR-J, Chen L, Goldenson J, Spetz J, Kawamura LM, 2012. Isoniazid vs. rifampin for latent tuberculosis infection in jail inmates: toxicity and adherence. $J$ Correct Health Care 18: 131-142.

11. Churchyard GJ, Fielding KL, Lewis JJ, Coetzee L, Corbett EL, Godfrey-Faussett P, Hayes RJ, Chaisson RE, Grant AD, 2014. A trial of mass isoniazid preventive therapy for tuberculosis control. N Engl J Med 370: 301-310.

12. Carbone AdaS et al., 2015. Active and latent tuberculosis in Brazilian correctional facilities: a cross-sectional study. BMC Infect Dis 15: 24.

13. Mabud TS et al., 2019. Evaluating strategies for control of tuberculosis in prisons and prevention of spillover into communities: an observational and modeling study from Brazil. PLoS Med 16: e1002737.

14. Comstock GW, Baum C, Snider DE, 1979. Isoniazid prophylaxis among Alaskan Eskimos: a final report of the Bethel isoniazid studies. Am Rev Respir Dis 119: 827-830.

15. Golub JE, Cohn S, Saraceni V, Cavalcante SC, Pacheco AG, Moulton LH, Durovni B, Chaisson RE, 2015. Long-term protection from isoniazid preventive therapy for tuberculosis in HIV-infected patients in a medium-burden tuberculosis setting: the TB/HIV in Rio (THRio) study. Clin Infect Dis 60: 639-645.

16. Babor TF, Higgins-Biddle J, Saunders JB, Monteiro M, 2001. The alcohol use disorders identification test: guideline for use in primary care. Braz J Probab Stat 17: 91-105.

17. Nemes $E$ et al., 2017. Optimization and interpretation of serial QuantiFERON testing to measure acquisition of Mycobacterium tuberculosis infection. Am J Respir Crit Care Med 196: 638-648.

18. Andrews JR et al., 2017. Serial QuantiFERON testing and tuberculosis disease risk among young children: an observational cohort study. Lancet Respir Med 5: 282-290.

19. Winje BA et al., 2018. Stratification by interferon- $y$ release assay level predicts risk of incident TB. Thorax 73: 652-661.

20. Abraham J, 2010. International conference on harmonisation of technical requirements for registration of pharmaceuticals for human use. Tietje C, Brouder A, eds. Handbook of Transnational Economic Governance Regimes. Leiden, The Netherlands: Brill|Nijhoff.

21. Common Terminology Criteria for Adverse Events (CTCAE)|Protocol Development|CTEP, 2017. Available at: https://ctep.cancer. gov/protocolDevelopment/electronic_applications/ctc.htm\#ctc_40. Accessed September 13, 2019.

22. Salpeter SR, 1993. Fatal isoniazid-induced hepatitis. Its risk during chemoprophylaxis. West J Med 159: 560-564.

23. Saukkonen JJ et al., 2006. An official ATS statement: hepatotoxicity of antituberculosis therapy. Am J Respir Crit Care Med 174: 935-952.

24. Ferebee SH, 1970. Controlled chemoprophylaxis trials in tuberculosis. A general review. Bibl Tuberc 26: 28-106.

25. Egsmose T, Ang'awa JOW, Poti SJ, 1965. The use of isoniazid among household contacts of open cases of pulmonary tuberculosis. Bull World Health Organ 33: 419-433.
26. Slater ML, Welland G, Pai M, Parsonnet J, Banaei N, 2013. Challenges with QuantiFERON-TB Gold assay for large-scale, routine screening of U.S. healthcare workers. Am J Respir Crit Care Med 188: 1005-1010.

27. Nemes $E$ et al., 2018. Prevention of $M$. tuberculosis infection with $\mathrm{H} 4$ :IC31 vaccine or BCG revaccination. N Engl J Med 379: 138-149.

28. Andrews JR, Hatherill M, Mahomed H, Hanekom WA, Campo M, Hawn TR, Wood R, Scriba TJ, 2015. The dynamics of QuantiFERON-TB Gold in-tube conversion and reversion in a cohort of South African adolescents. Am J Respir Crit Care Med 191: 584-591.

29. Dorman SE et al.; Tuberculosis Epidemiologic Studies Consortium, 2014. Interferon-y release assays and tuberculin skin testing for diagnosis of latent tuberculosis infection in healthcare workers in the United States. Am J Respir Crit Care Med 189: 77-87.

30. Gupta RK et al., 2019. Quantitative IFN-y release assay and tuberculin skin test results to predict incident tuberculosis. A prospective cohort study. Am J Respir Crit Care Med 201: 984-991.

31. Zwerling A, Benedetti A, Cojocariu M, McIntosh F, Pietrangelo F, Behr MA, Schwartzman K, Menzies D, Pai M, 2013. Repeat IGRA testing in Canadian health workers: conversions or unexplained variability? PLoS One 8: e54748.

32. Bastos ML, Menzies D, Belo MTCT, Teixeira EG, de Abreu ST, Antas PRZ, Trajman A, 2013. Changes in QuantiFERON@-TB Gold in-Tube results during treatment for tuberculous infection. Int J Tuberc Lung Dis 17: 909-916.

33. Alexander $W, 2013$. The uphill path to successful clinical trials. $P T$ 38: 225-227.

34. Little RJ et al., 2012. The prevention and treatment of missing data in clinical trials. N Engl J Med 367: 1355-1360.

35. Weber WW, Hein DW, 1979. Clinical pharmacokinetics of isoniazid. Clin Pharmacokinet 4: 401-422.

36. Farmanguinhos, 2017. Label of the Isoniazid. Available at: http://www2.far.fiocruz.br/farmanguinhos/images/stories/ phocadownload/isoniazida\%20100\%20e\%20300mg.pdf. Accessed May 7, 2016.

37. Isoniazid FDA Label - Tablet, 2016. AIDSinfo. Available at: https:// aidsinfo.nih.gov/drugs/123/isoniazid/11/professional. Accessed September 13, 2019.

38. Menzies $D$ et al., 2018. Four months of rifampin or nine months of isoniazid for latent tuberculosis in adults. N Engl J Med 379: 440-453.

39. Fresard I, Bridevaux PO, Rochat T, Janssens JP, 2011. Adverse effects and adherence to treatment of rifampicin 4 months vs isoniazid 6 months for latent tuberculosis: a retrospective analysis. Swiss Med Wkly 141: w13240.

40. Belknap R et al.; TB Trials Consortium iAdhere Study Team, 2017. Self-administered versus directly observed once-weekly isoniazid and rifapentine treatment of latent tuberculosis infection: a randomized trial. Ann Intern Med 167: 689-697.

41. Goldman AL, Braman SS, 1972. Isoniazid: a review with emphasis on adverse effects. Chest 62: 71-77.

42. Kunst $\mathrm{H}$, Khan KS, 2010. Age-related risk of hepatotoxicity in the treatment of latent tuberculosis infection: a systematic review. Int J Tuberc Lung Dis 14: 1374-1381. 